\title{
Cell-Free Layer (CFL) Analysis in a Glass Capillary: Comparison Between a Manual and Automatic Method
}

\author{
David Bento*, Diana Pinho****, Ana I. Pereira*,\$ and Rui Lima**** \\ ${ }^{*}$ PolytechnicInstituteof Bragança, ESTiG/IPB, Bragança, Portugal. \\ **CEFT, Faculdade de Engenharia da Universidade do Porto (FEUP), Porto, Portugal.

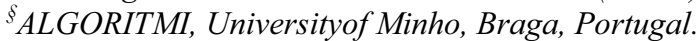

\begin{abstract}
In this study, in vitro blood flowing through a $100 \mu \mathrm{m}$ glass capillary was studied. The images were captured using a confocal system and post-processed using Image $J$ and MatLab. The aim of the present work, was to measure the trajectories of the cell-free layer (CFL) by using two different methods, i. e., a manual method (MM) and an automatic method (AM). For theMM we have used amanual tracking plugin (MTrackJ) from Image $J$ to track labeled red blood cells (RBCs) flowing around the boundary of the RBCs core. For the AM we have used a MatLab scripts to measure automatically the CFL trajectories. The preliminary numerical results suggest that the CFL trajectories follow a polynomial function for both methods.
\end{abstract}

Keywords: Cell-free layer.Automatic Method.Red Blood Cells.Nonlinear Optimization. PACS: $02.60 . \mathrm{Pn}$

\section{INTRODUCTION}

Awell known hemodynamic phenomenon observed in both in vivo and in vitro studies states that for narrow microtubes $(<300 \mu \mathrm{m})$ both hematocrict and apparent blood viscosity decreases as the tube diameter is reduced [1, 2].The physical reason behind this phenomenon, known as Fahraeus-Lindqvist effect, is the tendency of RBCs to migrate toward the centre of the microtube resulting in a marginal cell-free layer (CFL) at regions adjacent to wall [3].Recently, studies have shown strong evidence that the formation of the CFL is affected by both the geometry of the microchannel $[4,5,6,7]$ and the physiological conditions of the working fluid $[8,9]$.Although there have been several studies on the measurement of CFL thickness[10,11], according to our knowledge there have been very few studies on the determination of CFL trajectory. The main purpose of the present work is to measure several trajectories of the CFL by means of a manual method (MM) and an automatic method (AM) in a $100 \mu \mathrm{m}$ glass capillary. This experimental study was performed using a confocal microscopy system together with image analysis techniques. Additionally, nonlinear optimization techniques were used to compare automatic and manual methods.

\section{MATERIALS AND METHODS}

\section{Working fluids and microchannel geometry}

The working fluid used in this study was Dextran 40 (Dx-40;Otsuka Medicine) containing 12 $2 \%$ (12Hct) of human RBCs. The Hctscorresponded to the feed reservoirHct and were measured using a hematocrit centrifuge (Kubota 3220)immediately before each experiment. The RBCs were labeled with a lipophilic carbocyanime derivative, chloromethylbenzamido (CM-Dil, C-7000, Molecular Probes). A detailed description about the procedure for labeling the human RBCs can be found elsewhere [12]. The capillaries tested in this study were 100$\mu \mathrm{m}$ circular borosilicate glass microchannel fabricated by Vitrocom (Mountain Lakes). The capillary was mounted on a slide glass was immersed in glycerol to minimize the refraction from the walls.

\section{Experimental set-up}

The confocal micro-PTV system used in this study consists of an inverted microscope (IX71; Olympus) combined with a confocal scanning unit (CSU22; Yokogawa), a diode-pumped solid-state (DPSS) laser (Laser Quantum) with an excitation wavelength of $532 \mathrm{~nm}$ and a high-speed camera (Phantom v7.1; Vision Research). The

Numerical Analysis and Applied Mathematics ICNAAM 2012

AIP Conf. Proc. 1479, 786-789 (2012); doi: 10.1063/1.4756255

(C) 2012 American Institute of Physics 978-0-7354-1091-6/\$30.00 
glass capillarywas placed on the stage of the inverted microscope and by using a syringe pump (KD Scientific) a pressure-driven flow was kept constant. Additionally, by using a thermo plate controller (Tokai Hit) the temperature was set to $37^{\circ} \mathrm{C} \pm 1$. More detailed information about this system can be found elsewhere [5, 12, 13].Figure 1 shows the blood samples preparation and experimental set-up used in this study.

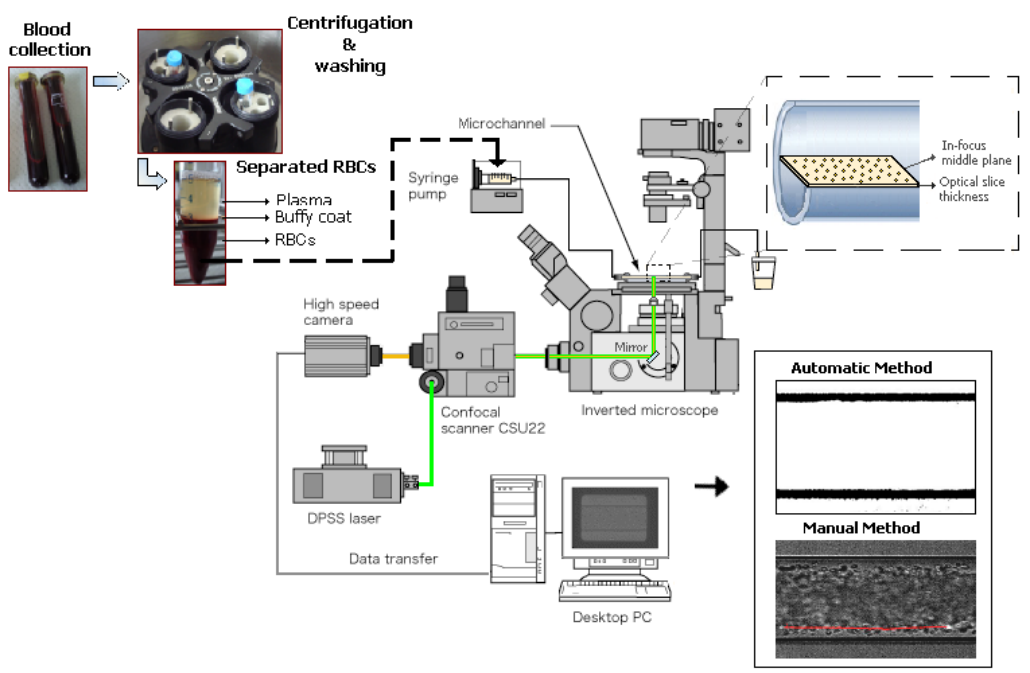

FIGURE 1.Blood samples preparation and experimental set-up.

The confocal images were captured around the middle of the capillary with a resolution of $640 \times 480$ pixel at a rate of 100 frames/s. Two image analyses methods were used in this study: manual method (MM) and automatic method (AM).

\section{Manual Method (MM)}

A manual tracking plugin (MTrackJ) [14] of an image analysis software (Image J, NIH) [15] was used to track the label RBCs. By using MTrackJ plugin, the bright centroid of the selected RBC was used through successive images. After obtaining series of $x$ and $y$ positions, data were exported for MatLab and the cftool package was used to calculate the best function that fits the experimental data.Figure 2 shows a trajectory of a labeled RBC flowing around the boundary region between the CFL and RBCs core.
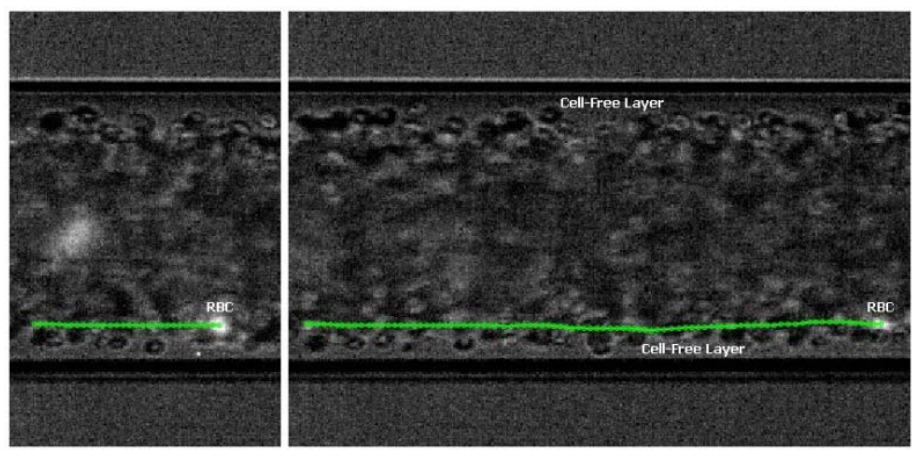

FIGURE 2.RBC trajectory, at different time intervals, flowing around the boundary region between CFL and RBCs core.

\section{Automatic Method (AM)}

Theframeswere processed usingimage processingtechniquesin MatLab [16]. Firstly, it wasapplied to eachframeamedian filterto removethe noise from the images, by usinga mask $3 \times 3$ pixel. Then the intensityof each pixel inthe frames sequence was evaluated to obtainan image with themaximumintensity. As a final step, the 
previously image (Fig. 3b) was convertedinto a binary image (Fig. 3c). Finally, the region of interest is selected and the upper and lower CFL trajectories are automatically measured.Figure 3 shows the image processingsequence to obtain the resulting binary image.
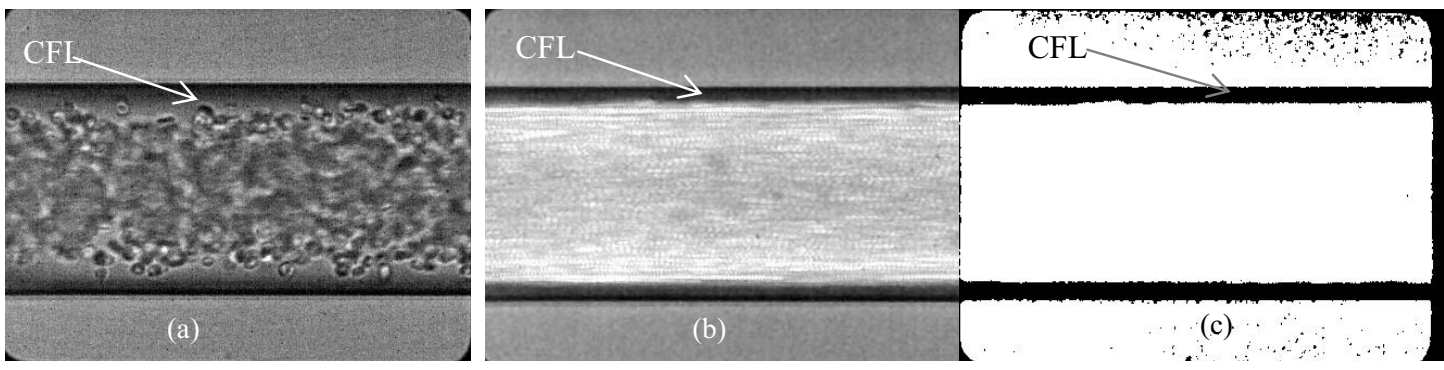

FIGURE 3.a) Original image; b) Maximum intensity image; c) Binary image.

\section{RESULTS AND DISCUSSION}

After obtaining series of $x$ and $y$ positions for both methods, data were exported for the determination of each individual CFL trajectory and to analyze the best mathematical function that approximates to the CFLs experimental flow behavior.

In this study it was measured and analyzed two CFLs trajectories at a temperature of $25{ }^{\circ} \mathrm{C}$.For each CFL,by using the MTrackJ plugin (MM) form ImageJ and by using the proposedautomatic method (AM), we have obtained $\left\{\left(x_{j}, y_{j}\right), j=1, \ldots, k\right\}$ data. To compare the consistency of both methods we decided to determine the better approximation for the data by usingthe tool cftoolexisting in the Curve Fitting Toolbox fromMatLab[17].

Hence,we consider seven different functions: polynomials, exponential, Fourier and power functions.

The error of nonlinear least squares approximation of twoCFLs trajectories are listed in the Table 1, where $F_{l}=\sum_{j=1}^{k}\left(y_{j}-f_{l}\left(x_{j}\right)\right)^{2}$ is the nonlinear least squares approximation error of the function $f_{l}(x)$, with $l=1, \ldots, 7$.

TABLE 1. Error of the seven best functions obtained with cftool.

\begin{tabular}{c|cc|cc}
\hline Function & \multicolumn{2}{|c|}{$\boldsymbol{F}_{\boldsymbol{l}}$ - Manual } & \multicolumn{2}{c}{$\boldsymbol{F}_{\boldsymbol{r}}$ Automatic } \\
\hline Poly5 & $\mathbf{1 8 . 0 6 0 6}$ & $\mathbf{1 8 . 6 3 2 7}$ & $\mathbf{1 7 . 3 7 1 7}$ & $\mathbf{1 2 . 5 8 7 7}$ \\
Poly4 & 20.2669 & 22.9811 & 18.4294 & 15.7613 \\
Poly3 & 24.1842 & 23.7068 & 20.0685 & 16.1108 \\
Poly2 & 25.7616 & 23.7738 & 20.4083 & 16.1177 \\
Exp2 & 24.6697 & 23.7505 & 20.2906 & 16.1024 \\
Fourier1 & 20.2669 & 21,6219 & 23.1763 & 16.6979 \\
Power2 & 51.3068 & 24.3397 & 20.5599 & 15.7486 \\
\hline
\end{tabular}

The results from Table 1 suggest that the best mathematical function that fits thedata is based on the polynomial of degree five.

\section{CONCLUSIONS}

The present study indicates that the CFL trajectories that exist in microchannels, follow a polynomial function of degree five. Additionally, the data obtained from the proposed automatic method (AM)is equivalent todata obtained from themanual method (MM). Hence, the proposed automatic method is a promising way to measure automatically the trajectory of the CFL in microchannels. 


\section{ACKNOWLEDGMENTS}

The authors acknowledge the financial support provided by: PTDC/SAU-BEB/108728/2008, PTDC/SAUBEB/105650/2008, PTDC/EME-MFE/099109/2008 andPTDC/SAU-ENB/116929/2010 from the FCT (Science and Technology Foundation) and COMPETE, Portugal.

\section{REFERENCES}

1. A. Pries, D. Neuhaus, P. Gaehtgens, Am J Physiol263, H1770-H1778(1992).

2. H. Goldsmith, G. Cokelet, P. Gaehtgens, Am. J. Physiol. 257, H1005-H10015 (1989).

3. C. Caro, T. Pedley, R. Schroter, W. Seed, The mechanics of the circulation, Oxford University Press, 1978.

4. D. Pinho, Determination and characterization of red blood cells trajectories: a semi-automatic method, Master in Biomedical Technology, Polytechnic Institute of Bragança, Portugal, 2011 (in portuguese).

5. R. Lima, S. Wada, S. Tanaka, M. Takeda, T. Ishikawa, K. Tsubota, Y. Imai, Y. Yamaguchi,BiomedicalMicrodevices, 10(2), 153-167 (2008).

6. R. Lima, T. Ishikawa, Y. Imai,M. Takeda, S. Wada, T. Yamaguchi,Journal of Biomechanics41, 2188-2197 (2008).

7. V. Leble, R. Lima, R. P. Dias, C. S. Fernandes, T. Ishikawa, Y. Imai, T. Yamaguchi,Biomicrofluidics5, 044120 (2011).

8. H. Fujiwara, T. Ishikawa, R. Lima, N. Matsuki, Y. Imai, H. Kaji, M. Nishizawa, T. Yamaguchi,Journal of Biomechanics42, 838-843(2009).

9. V. Garcia, R. Dias, R. Lima, In Vitro Blood Flow Behaviour in Microchannelswith Simple and Complex Geometries, Applied Biological Engineering - Principles and Practice, Ganesh R. Naik(ed.), InTech, 17, 394-416, 2012.

10. B. Namgung, P. K. Ong, Y. H. Wong, D. Lim, K. C. Chun, S. Kim, Physiological Measurement31: N61-N70 (2010).

11. S. Kim, R. L. Kong, A. S. Popel, M. Intaglietta, P. C. Jonhson, Microcirculation13:199-207 (2006).

12. R. Lima, T. Ishikawa, Y. Imai, M. Takeda, S. Wada, T. Yamaguchi,Annals of Biomedical Engineering. 37, 1546-59(2009).

13. R. Lima, S. Wada, K. Tsubota,T. Yamaguchi,MeasurementScience and Technology17, 797-808(2006).

14. E. Meijering, I. Smal and G. Danuser, IEEE Signal Process. Mag. 23: 46-53(2006).

15. M. Abramoff, P. Magelhaes and S. Ram, Biophotonics Int. 11: 36-42(2004).

16. S. L. Eddins. R. C. Gonzalez, R. E. Woods, Digital Image Processing Using Matlab, 2002.

17. MathWorks, Optimization Toolbox User's Guide, 2010. 